\title{
Guest Editorial: Special Issue on Network and Parallel Computing for Emerging Architectures and Applications
}

\author{
Feng Zhang ${ }^{1} \cdot$ Jidong Zhai ${ }^{2} \cdot$ Marc Snir $^{3} \cdot$ Hai Jin ${ }^{4} \cdot$ Hironori Kasahara ${ }^{5}$. \\ Mateo Valero ${ }^{6}$
}

Published online: 23 March 2019

๑) Springer Science+Business Media, LLC, part of Springer Nature 2019

The aim of this special issue is to provide an introduction to the state-of-the-art technology and challenges of the High Performance Computing and Big Data areas. This special issue consists of 12 papers that are briefly discussed as follows.

This special issue has three papers that relate to deep neural networks, "FloatFix: An Efficient and Hardware-Friendly Data Type for Deep Neural Network", "BSHIFT: A Low Cost Deep Neural Networks Accelerator", and "Training Deep Nets with Progressive Batch Normalization on multi-GPUs".

There are three algorithm papers for accelerating kernels or applications on emerging architectures, "ASW: Accelerating Smith-Waterman Algorithm on Coupled CPU-GPU Architecture", "Register-Aware Optimizations for Parallel Sparse Matrix-Matrix Multiplication", "Optimizing Sparse Matrix-Vector Multiplications on An ARMv8-based Many-Core Architecture".

Three papers relate to architecture and networking, "HARE: History-Aware Adaptive Routing Algorithm for Endpoint Congestion in Networks-on-Chip", "Lightweight and Accurate Memory Allocation in Key-value Cache", and "Improving the Performance of Distributed MXNet with RDMA".

The other three papers are system papers, "Migration cost and energy-aware virtual machine consolidation under cloud environments considering remaining runtime", "A Dependency-Aware Storage Schema Selection Mechanism for In-Memory Big Data Computing Frameworks", and "ElasticActor: An Actor System with Automatic Granularity Adjustment".

Feng Zhang

fengzhang@ruc.edu.cn

1 Renmin University of China, Beijing, China

2 Tsinghua University, Beijing, China

3 University of Illinois at Urbana-Champaign, Urbana, IL, USA

4 Huazhong University of Science and Technology, Wuhan, China

5 Waseda University, Shinjuku-ku, Japan

6 Barcelona Supercomputing Center, Barcelona, Spain 
The special issue was preceded by the 15th Annual IFIP International Conference on Network and Parallel Computing (November 29-December 1, 2018, in Muroran, Hokkaido). The papers have undergone rigorous peer-review according to the journal's high standards. These 12 contributions encompass a wide range of research and topics, thereby appealing to both the experts in the field and those who want a snapshot of the current breadth of HPC and big data research.

We would like to thank everybody who contributed to the special issue.

Enjoy reading,

Guest Editors:

Feng Zhang

Jidong Zhai

Marc Snir

Hai Jin

Hironori Kasahara

Mateo Valero

August 2018

Publisher's Note Springer Nature remains neutral with regard to jurisdictional claims in published maps and institutional affiliations. 